\title{
Continuous time estimation as a behavioural index of human cerebral ischaemia during temporary occlusion of the internal carotid artery
}

\author{
Ronald M Lazar, Randolph S Marshall, John Pile-Spellman, William L Young, \\ Lotfi Hacein-Bey, Richard P Sloan, J P Mohr
}

\begin{abstract}
Objective-To determine whether a continuous time estimation task during test occlusions of either internal carotid artery would increase the ability to detect the earliest signs of cerebral ischaemia in the anterior circulation.

Methods-Four patients were involved in real time measurement of their timing accuracy before, during, and after each test occlusion. While under each test condition, patients were instructed to press a mouse button connected to a computer and then to press it again no sooner than 10 seconds from the previous response but no longer than 13 seconds later. While being given automated feedback on accuracy, patients were instructed to continually press the mouse on the target schedule to maximise correct responses until told to stop.
\end{abstract}

Results-The data showed deterioration of timing accuracy during carotid occlusion $(P<0.05)$, which always preceded the onset of physical signs and correlated in one patient with the presence of reduced regional cerebral blood flow.

Conclusion-Decline of sustained attention under conditions of test balloon occlusion of either internal carotid artery was an indicator of failure to maintain adequate cerebral blood flow to sustain normal neurological function. The demonstration of the behavioural effects of early cerebral ischaemia shows the feasibility of an experimental model for the study of human brain function, and may now make it possible to quantify more precisely the time course of acute ischaemic events.

(F Neurol Neurosurg Psychiatry 1996;60:559-563)

Keywords: interresponse times; continuous performance; cerebral ischaemia; carotid occlusion

The normal brain maintains adequate blood flow via autoregulation by altering the size of cerebral vessels to compensate for changes in systemic arterial blood pressure. ${ }^{1}$ Cerebral ischaemia occurs beyond the point when the upper limits of oxygen extraction have been reached. ${ }^{2}$ The determination of the point at which ischaemic neurons begin losing their functional capacity has been established mainly with animal models because of the obvious complexities associated with experi- mental study of cerebral ischaemia in humans. We report the feasibility of the scientific study of cerebral ischaemia in patients using behavioural methods in the setting of test balloon occlusions of the carotid artery. Patients with head and neck tumours involving the carotid artery, as well as those with inoperable carotid cavernous aneurysms, are sometimes treated by internal carotid occlusion. ${ }^{3}$ Typically, patients undergo short lived, test occlusions of the carotid artery with a balloon tipped catheter to evaluate the consequences of anticipated surgery or permanent occlusion. ${ }^{456}$ In most centres where test occlusions are performed, there is some concurrent assessment of function, usually by routine neurological examination; some include transcranial Doppler ultrasonography or EEG, and even measurement of cerebral blood flow via injected ${ }^{133} \mathrm{Xe}^{3}$ Many patients tolerate carotid occlusion without symptoms. Others, however, develop neurological deficits when or shortly after the carotid is occluded, or during subsequent hypotension that is induced to test the limits of cerebrovascular reserve. Our objective was to determine whether concurrent measurement of behavioural function would increase our ability to discern any sign of brain dysfunction under potentially ischaemic conditions. To achieve the goal of establishing the time course of ischaemia, a behavioural index was chosen that would measure sustained attention and concentration, making our method similar in principle to the continuous performance test used in clinical neuropsychological assessment. ${ }^{7}$ The aim was to determine whether we could detect the earliest effects of ischaemia in the anterior circulation in either the left or right hemispheres.

Methods

PATIENTS

Four patients (three women and one man) were selected for study based on a clinical indication for a test occlusion: two received occlusion of the left internal carotid artery and two were occluded on the right. Informed consent for our procedure was obtained as part of the general consent for the carotid test occlusion procedure. Patient 1 , aged 48 , had a left carotid cavernous aneurysm, possibly traumatic in origin. She was neurologically normal. Patient 2, aged 57, had a left cavernous aneurysm with an associated sixth nerve palsy. She had been treated in June 1993 with endovascular embolisation with suboptimal obliteration of the aneurysm. Patient 3, aged 
Figure 1 The time estimation procedure over a 29 second interval in which a patient made four mouse responses. The target time between responses

(interresponse time) was

between 10 and 13 seconds.

The clock reset after every response.

Time estimation paradigm

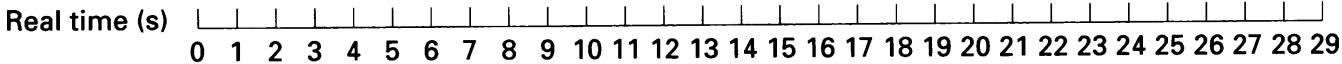

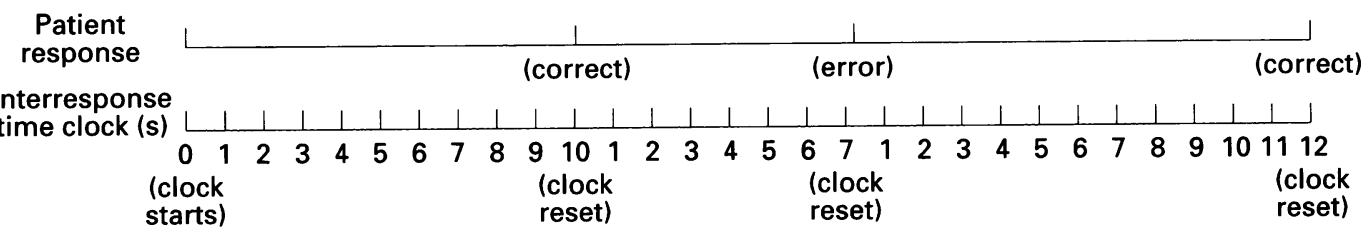

45, had a right cavernous aneurysm and had a normal neurological examination. Patient 4, aged 66, had a chest wall squamous cell carcinoma. This was treated with radiation, chemotherapy, and surgery, but recurred in the neck surrounding the right common carotid artery. He had right vocal cord paresis but no other neurological abnormalities.

\section{PROCEDURES}

Carotid test occlusions were performed after routine angiograms. A catheter with a balloon tip was inserted via the femoral artery into the target carotid artery so that the balloon tip was positioned in the extracranial portion of the internal carotid artery distal to the common carotid bifurcation. Mean arterial pressure was measured with a strain gauge from the femoral introducer sheath. Baseline neurological examination was performed. For patients 3 and 4, cerebral blood flow was measured by intracarotid ${ }^{133} \mathrm{Xe}$ injection with calculation of initial slope. The balloon was then inflated, pressures recorded, and a conventional neurological examination was performed again. If the patient was judged clinically stable, intravenous sodium nitroprusside was used to induce stages of increasing hypotension, with neurological examination between each hypotensive phase. The balloon was deflated either if the patient tolerated carotid occlusion under conditions of significant hypotension, or if the patient developed a gross neurological abnormality, such as hemiparesis or aphasia during the procedure. The following behavioural test was performed by the patient before, during, and after the test occlusion.

Patients were taught a time estimation procedure, using a Macintosh computer, as a measure of sustained attention and concentration. Before catheterisation, they were instructed to press a mouse button located at their right side underneath the bed sheet, and then to press it again no sooner than $10 \mathrm{sec}-$ onds but no longer than 13 seconds later than the previous response. The right hand was always used because of logistical considerations of the interventional neuroradiological suite. A correctly timed response resulted in a tone; a mistimed response produced a different sound. Each patient was told to continually press the mouse in this manner until told to stop. The "interresponse time" clock (measuring the time between consecutive mouse presses) was restarted after every response, and the patient was told to maximise the number of "correct" tones. Figure 1 illustrates the behavioural paradigm for four consecutive responses. Each patient underwent baseline behavioural testing after the insertion of the catheter but before balloon inflation, and then after inflation and under each hypotension stage. Patient timing was analysed with the SAS analysis of variance (ANOVA) program for Scheffe's multiple comparisons within the framework of the general linear model. ${ }^{8}$ Patients served as their own controls in an A-B-A (no treatment-treatment-no treatment) design.

\section{Results}

PATIENT 1

The upper graph in fig 2 shows patient 1's interresponse times over consecutive trials. With the catheter inserted into the left carotid artery but before inflation of the balloon, her mean time between responses ("interresponse time") was 13.84 (SD 4.39) seconds over two 23 trial blocks. About 70 seconds after the inflation of the balloon, her mean interresponse rose to 21.20 (SD 11.58) seconds, representing a significant decrease in accuracy and increase in variability compared with her baseline $(P<0.0001)$. A brief neurological examination (language, memory, and motor function) remained normal after the first 23 trial block under occlusion conditions. A second block of trials continued to show the same poor timing, but a second neurological examination showed her still normal. Her mean arterial pressure at the femoral artery was decreased from 103 to $63 \mathrm{~mm} \mathrm{Hg}$, and after two responses, her hand became hemiparetic and dropped the mouse. After the balloon was deflated and pressure returned to normal, a postocclusion assessment of her timing showed a return to values not different from her baseline (mean 10.75 (SD 2.63) s). Several days later, she underwent intracranialextracranial bypass surgery to improve collateral circulation. Two weeks later, she underwent another test occlusion with the time estimation test. The results of the behavioural measurement showed no significant change between her baseline performance and all subsequent phases of occlusion and induced hypotension.

PATIENT 3

This patient's mean timing performance during the pretreatment baseline over two trial blocks was 11.42 (SD 4.10) seconds. After occlusion of the right internal carotid artery with a mean arterial pressure of $110 \mathrm{~mm} \mathrm{Hg}$, there was no significant change in her mean 
Figure 2 Interresponse times for consecutive trials for two patients undergoing left internal carotid artery occlusion (above) and two patients receiving right internal carotid artery occlusions (below); MAP designates mean arterial pressure at the tip of the catheter in the femoral artery. The interval between adjacent blocks of trials was about five minutes.
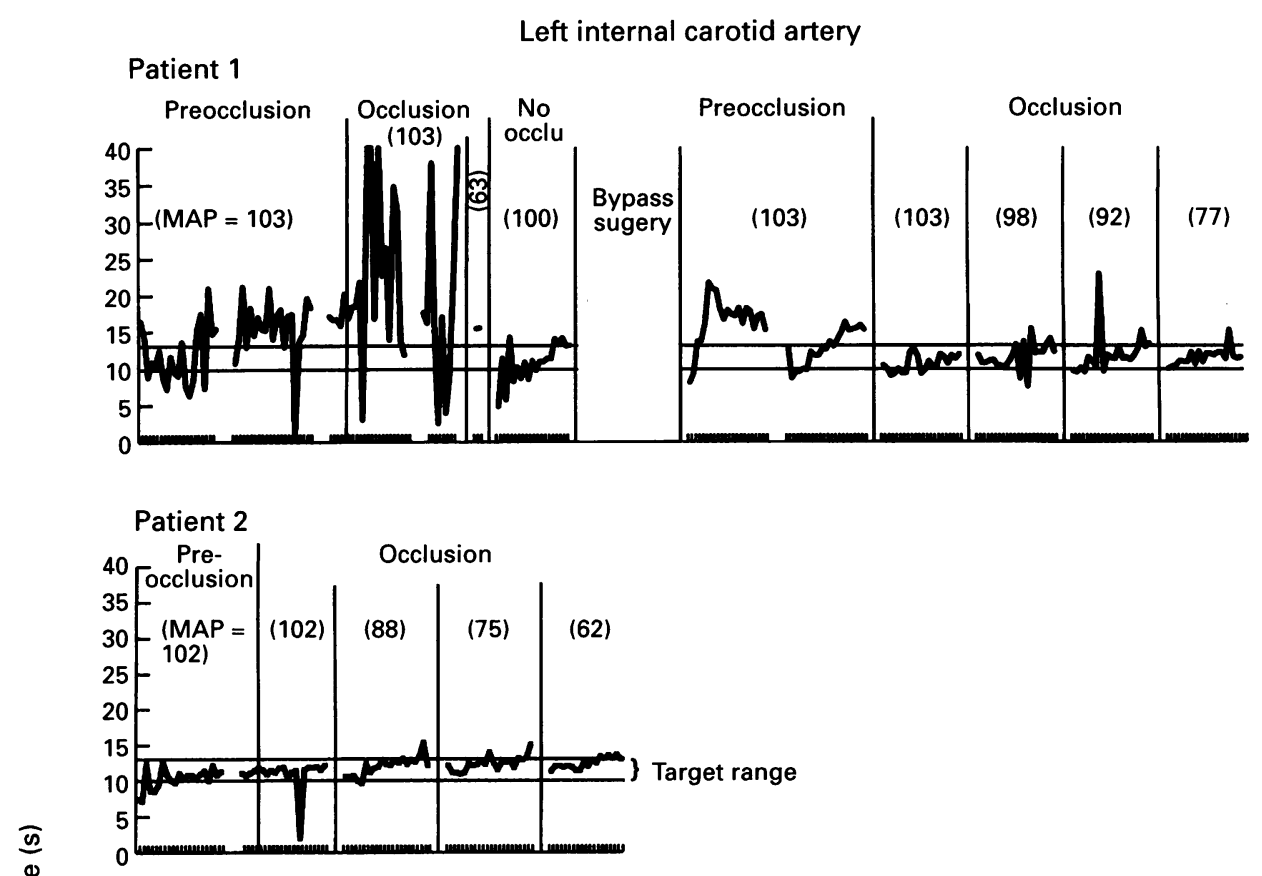

Right internal carotid artery

Patient 3
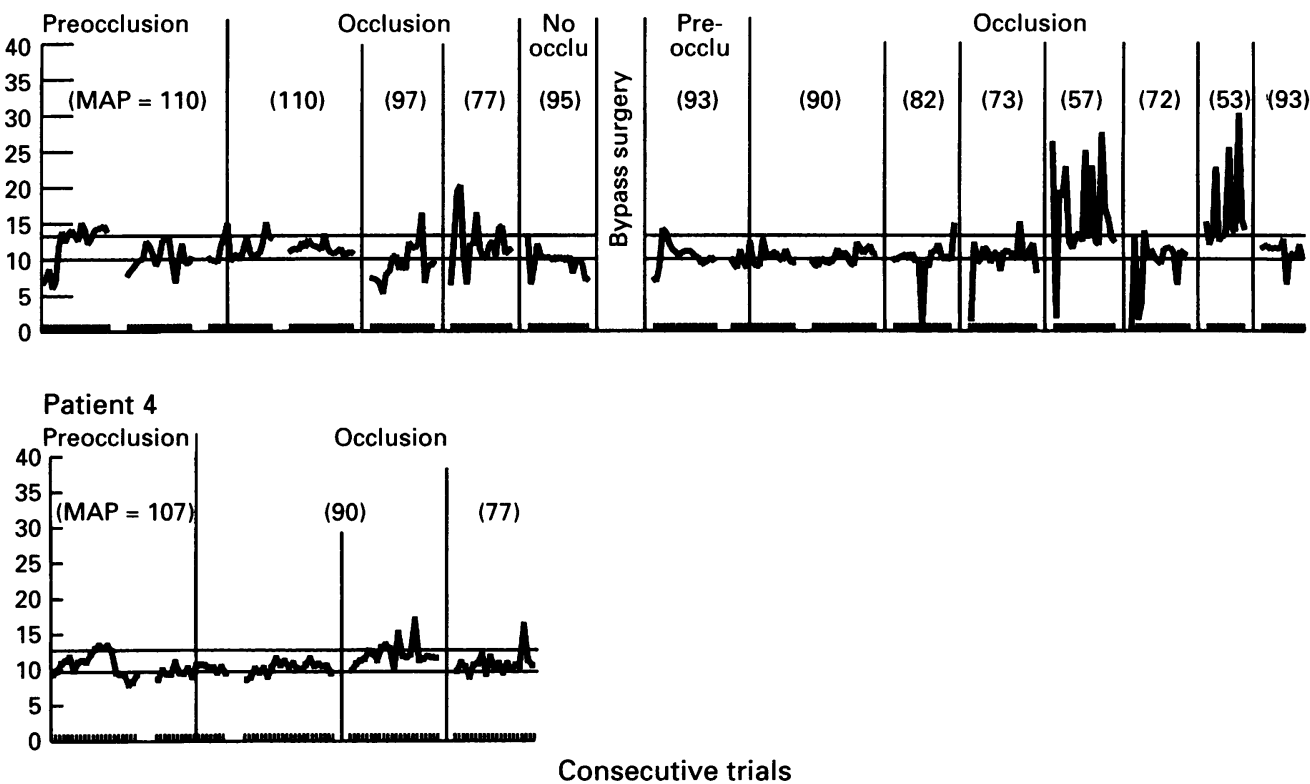

interresponse time performance $(11.11 \mathrm{~s})$ or response variability (SD $2 \cdot 46$ ), despite a $30 \%$ decline in regional cerebral blood flow compared with baseline flow. Her neurological examination was normal. During the first stage of induced hypotension at a mean arterial pressure of $97 \mathrm{~mm} \mathrm{Hg}$, her timing became significantly unstable $(P<0.05)$, but the neurological examination remained unchanged. A second decline in arterial pressure $(77 \mathrm{~mm}$ $\mathrm{Hg}$ ) was associated with a similar degree of instability of timing (mean $12 \cdot 28$ (SD $3 \cdot 48$ ) s), which was not significantly different from the previous block of trials. The balloon was deflated and mean arterial pressure was returned to $95 \mathrm{~mm} \mathrm{Hg}$ and a postocclusion block of trials showed a return to baseline values (mean 9.68 (SD 1.55) s). The patient then underwent intracranial-extracranial bypass surgery which failed one day later because of thrombosis in the bypass vessel. She had a second test occlusion, and again showed a $30 \%$ drop in regional cerebral blood flow. The patient's timing again deteriorated during significant hypotension, this time when her mean carotid arterial pressure was dropped to $57 \mathrm{~mm} \mathrm{Hg}$. At this point, her mean interresponse increased to $16 \cdot 17$ (SD 6.34) seconds $(\mathrm{P}<0.01)$. She was also found to have developed a mild central facial palsy and mild weakness in her left hand, not the one using the mouse. A return to the previous pressure $(72 \mathrm{~mm} \mathrm{Hg})$ showed a restabilisation of timing with no neurological deficit, but a second 
attempt at a lower pressure $(53 \mathrm{~mm} \mathrm{Hg})$ showed the same significant change in accuracy and variability of interresponse time with the concomitant neurological abnormalities. Her mean arterial pressure was returned to normal and her interresponse times were restored to baseline values (mean 11.01 (SD 1.48) s).

\section{PATIENT 2}

After occlusion of the left internal carotid, her interresponse times (mean 10.94 (SD 2.36) s) were not significantly different from her baseline (mean 10.43 (SD 1.37) s). There were no significant changes in accuracy during three phases of increasing hypotension. Her periodic neurological examinations were normal.

\section{PATIENT 4}

After occlusion of the right internal carotid, timing accuracy did not change from baseline (mean 10.54 (SD 1.56) s) to occlusion (mean $11.02(\mathrm{SD} 1.50) \mathrm{s}$ ), and there was no change after one stage of hypotension (mean $=10.83$ (SD 1.54) s). Because regional cerebral blood flow also showed less than a $5 \%$ change in perfusion, the patient was considered clinically able to tolerate the procedure and the balloon was deflated.

\section{Discussion}

Our results show that deterioration in time estimation under conditions of carotid test occlusion was an indicator of failure to maintain adequate blood flow to sustain normal neurological function, and appeared before any gross signs of clinical decline in function. In each case there was a deterioration of timing accuracy and increases in response variability which preceded the onset of physical signs and correlated with the presence of reduced regional cerebral blood flow. A cause and effect relation between haemodynamics and behaviour was shown by baseline evaluation and the decline in timing accuracy, followed by the immediate reversibility of the deficit by either deflating the balloon catheter or increasing blood pressure. Although there are behavioural paradigms of animals undergoing transient cerebral ischaemia, ${ }^{9}$ there has not yet been, to our knowledge, such an experimental behavioural model in humans. In the case of patient 1 , balloon occlusion alone was sufficient to induce behavioural deterioration as measured by alterations of interresponse times. Patient 3 did not show a decline in timing accuracy until both her carotid was blocked and hypotension reached an apparent threshold level. It is not clear whether our findings are attributable to alterations in timing, attention, or some other non-specific effect of hemispheric ischaemia. Hemiparesis, sensory loss, or apraxia cannot account for the effects in these two patients because the mouse was in the right hand of both patients yet the contralateral internal carotid was occluded for patient 1 and the ipsilateral side was occluded for patient 3 . The magnitude of the effect may have been enhanced for patient 1 because of the combined effects on behavioural and motor function.

It is possible that non-specific medication effects may have exaggerated the neurological effects of the occlusion. Nevertheless, the demonstration of stable timing behaviour before occlusion and the immediate return to comparable interresponse times immediately after deflation of the balloon showed that blocking the internal carotid was a necessary condition for the deterioration of timing. We empirically demonstrated control for the effects of such variables as the idiosyncratic effects of medication and neurovascular architecture when the patient's timing returned to normal values. ${ }^{10}$

The most common method of monitoring the status of brain function during balloon test occlusions has traditionally been the neurological examination, ${ }^{4}{ }^{11}$ but recent data have suggested that clinical evaluation alone is unreliable in identifying patients with diminished perfusion reserve. ${ }^{12}$ These same investigators also found that brain SPECT was not predictive of outcome from occlusion when patients went on to surgery. ${ }^{133} \mathrm{Xe}$ cerebral blood flow is also often used during test occlusions, ${ }^{311}$ but Yonas et al found no statistically significant difference in complication rates with internal carotid artery sacrifice in patients having prior test balloon occlusions alone versus occlusions with cerebral blood flow analysis. ${ }^{13}$

The use of continuous time estimation as a measure of temporal discrimination was initially explored as an operant conditioning procedure with animals. ${ }^{14}$ One common application in non-human species has been the study of the effects of drugs, such as amphetamines, ${ }^{15}$ lithium, ${ }^{16}$ and antidepressants. ${ }^{17}$ Similarly, investigations with humans have involved the evaluation of the effects of chronic alcoholism..$^{18}$ These techniques, however, have never been used to study abrupt changes in cerebral function in humans or applied to acute medical problems. Our demonstration that it is feasible to measure the behavioural effects of early cerebral ischaemia may now make it possible to quantify more precisely the time course of the neurological effects arising from decreases in blood flow to the brain.

1 Welch KMA, Levine SR. Focal brain ischemia and stroke: pathophysiology and acid-base status. In: Bornstein RA, pathophysiology and acid-base status. In: Bornstein RA, Brown G, eds. Neurobehavioral aspects of cerebrovascular disease. New York: Oxford University Press, 1991:17-38.
Pulsinelli WA, Jacewicz M. Animal brain models of cerePulsinelli WA, Jacewicz M. Animal brain models of cere-
bral ischemia. In: Barnett HJM, Mohr JP, Stein BM, Yatsu FM, eds. Stroke: pathophysiology, diagnosis and management. New York: Churchill-Livingstone, 1992 49-68.

3 Young WL, Pile-Spellman J. Anesthetic considerations for interventional neuroradiology. Anesthesiology 1994;80 427-56.

4 Berenstein A, Ransahoff J, Kupersmith J, Flamm E, Graeb D. Transvascular treatment of giant aneurysms of the cavernous carotid and vertebral arteries. Surg Neurol 1984;21:3-12.

5 Fox AJ, Vinuela F, Pelz DM, et al. Use of detachable balloons for proximal artery occlusion in the treatment of unclippable aneurysms. $\mathcal{F}$ Neurosurg 1987;66:40-6.

6 Mathis JM, Barr JD, Jungreis CA, et al. Temporary balloon occlusion of the internal carotid artery: experience in 500 occlusion of the internal carotid artery: experience

7 Lezak M. Neuropsychological assessment: 3rd ed. New York: Oxford University Press, 1995. 
8 SAS/STAT user's guide, version 6. 4th ed. Cary, IN: SAS Institute, 1990.

9 Willson RJ, Szkely AD, Stewart MG. Transient cerebra ischemia disrupts performance on a one-trial passive avoidance task in the domestic chick and is associated with neuronal degeneration in the central nervous system. Neurosci Res 1994;61:975-81.

10 Sidman M. Tactics of scientific research. New York: Basic Books, 1960.

11 Anon VV, Aymard A, Gobin YP, et al. Balloon occlusion of the internal carotid artery in 40 cases of giant cavernous aneurysm: technical aspects, cerebral monitoring, and aneurysm: technical aspects, cerebral

12 Lorberboym M, Machac J, Pandit N, et al. Predictive value Lorberboym M, Machac J, Pandit N, et al. Predictive value
of brain perfusion SPECT during temporary balloon occlusion of the internal carotid artery for perioperative complications. F Nucl Med 1995;5:539.

13 Yonas $\mathrm{H}$, Lindkey $M$, Johnson DW, et al. Internal carotid balloon test occlusion does require quantitative $\mathrm{CBF}$ AfNR Am $\mathcal{F}$ Neuroradiol 1992;13:1147-8.
14 Skinner BF. Schedules of reinforcement. New York: Appleton-Century, 1957.

15 Michaelis RC, Holloway FA, Bird DC, Huerta PI Interactions between stimulants: effects on DRL performance and lethality in rats. Pharmacol Biochem Behav 1987;27:299-306.

16 Harrigan T, Peredery O, Persinger MA. Failure to acquire an inhibitory task following seizure-induced brain damge. Percept Mot Skills 1990;70:268-70.

17 O'Donnell JM, Seiden LS. Effect of the experimental antidepressant ARH-9377on performance during differential reinforcement of low response rate. Psychopharmcology reinforcement of

18 Davidson RS, Bremser RF. Controlled alcoholic drinking: differential reinforcement of low rates of drinking. Behav Modif 1977;1:221-34

19 Oscar-Berman M, Zola-Morgan SM, Oberg RGE, Bonner RT. Comparative neuropsychology and Korsakoff's syndrome. III. Delayed response, delayed alternation and DRL performance. Neuropsychologia 1982;20:187-202.

\section{NEUROLOGY IN LITERATURE}

\section{More movements}

Descriptions of parkinsonian patients are surprisingly infrequent in literature. Dickens' character, in The Pickwick papers, exhibits immobility with tremor, and is probably parkinsonian rather than depressed. Some of the Witches of Salem seem to have had oculogyric crises, raising the question of an encephalitic basis for their illness. Constance, in the Old wives' tale, appears to have developed late-onset chorea as does Dick, in The Heart of Midlothian. Uriah Heep's serpiginous movements are not, I suspect, the result of Dickens observing athetosis, but his attempt to portray the evil, serpent-like, characteristics of that individual. Claudius, one of Suetonius' twelve Caesars, probably had multiple tics, but without the vocal utterances that would establish a diagnosis of Gilles de la Tourette's syndrome.

Sir Walter Scott, 1830, The Heart of Midlothian

When Dick had looked at the paper, he winked with one eye, extended his grotesque mouth from ear to ear, like a navigable canal, scratched his head powerfully ... He ducked with his head and shoulders, scraped with his more advanced hoof, bolted the alcohol, to use the learned phrase, and withdrew to his own domains.

Charles Dickens, 1836-7, The Pickwick papers On the opposite side of the room an old man was seated on a small wooden box, with his eyes rivetted on the floor, and his face settled into an expression of the deepest and most hopeless despair. A young girlhis little grand-daughter-was hanging about him: endeavouring, with a thousand childish devices, to engage his attention; but the old man neither saw nor heard her. The voice that had been music to him, and the eyes that had been light, fell coldly on his senses. His limbs were shaking with disease, and the palsy had fastened on his mind.
Charles Dickens, 1849-50, David Copperfield

He had a way of writhing when he wanted to express enthusiasm, which was very ugly; and which diverted my attention from the compliment he had paid my relation, to the snaky twistings of his throat and body ... Uriah, with his long hands slowly twining over one another, made a ghastly writhe from the waist upwards, to express his concurrence in this estimation of me. ... He jerked himself about, after this compliment, in such an intolerable manner, that my aunt, who had sat looking straight at him, lost all patience. "Deuce take the man!" said my aunt, sternly, "What's he about? Don't be galvanic, sir!"

Arnold Bennett, 1908, The old wives' tale Constance, for all her vast bulk, continually made little nervous movements. Occasionally she would sniff and occasionally a mysterious noise would occur in her chest; she always pretended that this noise was a cough, and would support the pretence by emitting a real cough immediately after it.

Gaius Suetonius, The twelve Caesars; Tranquillus (translated by Robert Graves, 1957)

These included an uncontrolled laugh, a horrible habit, under the stress of anger, of slobbering at the mouth and running at the nose, a stammer, and a persistent nervous tic-which grew so bad under emotional stress that his head would toss from side to side.

Folio Society, 1982, The witches of Salem

$\ldots$ and then her eyes were presently pulled into her head, so farr, that one might, have fear'd she should never have us'd them more.

$$
\begin{array}{r}
\text { G D PERKIN } \\
\text { Regional Neurosciences Centre } \\
\text { Charing Cross Hospital } \\
\text { Fulham Palace Road } \\
\text { London W6 } 8 R F, U K
\end{array}
$$

